\title{
Effects of Red Grape Juice Consumption on High Density Lipoprotein- Cholesterol, Apolipoprotein AI, Apolipoprotein B and Homocysteine in Healthy Human Volunteers
}

\author{
Mohammad H. Khadem-Ansari*, Yousef Rasmi and Fatemeh Ramezani
}

Department of Clinical Biochemistry and Nutrition, Faculty of Medicine, Urmia University of Medical Sciences, Urmia, Iran

\begin{abstract}
It has suggested that grape juice consumption has lipid- lowering effect and it is associated with a decreased risk of heart disease. We aimed to evaluate the effects of red grape juice (RGj) consumption on high density lipoproteincholesterol (HDL-C), apolipoprotein AI (apoAI), apolipoprotein B (apoB) and homocysteine (Hcy) levels in healthy human volunteers. Twenty six healthy and nonsmoking males, aged between 25-60 years, who were under no medication asked to consume $150 \mathrm{ml}$ of RGj twice per day for one month. Serum HDL-C, apoAI, apoB and plasma Hcy levels were measured before and after one month RGj consumption. HDL-C levels after RGj consumption were significantly higher than the corresponding levels before the RGj consumption (41.44 \pm 4.50 and $44.37 \pm 4.30 \mathrm{mg} / \mathrm{dl} ; \mathrm{P}<0.0001)$. Also, apoB was significantly increased after RGj consumption (149.0 \pm 22.35 and $157.19 \pm 18.60 \mathrm{mg} / \mathrm{dl} ; \mathrm{P}<0.002)$. But apoAI levels were not changed significantly before and after of RGj consumption (154.27 \pm 21.55 and $155.35 \pm 21.07 \mathrm{mg} / \mathrm{dl} ; \mathrm{P}>0.05)$. Hcy levels were decreased after RGj consumption $(7.70 \pm 2.80$ and $6.20 \pm 2.30 \mu \mathrm{mol} / \mathrm{l} ; \mathrm{P}<0.001)$. The present study demonstrates that $\mathrm{RGj}$ consumption can significantly increase serum HDL-C levels and decrease Hcy levels. These findings may have important implications for the prevention of atherosclerosis in healthy individuals.
\end{abstract}

Keywords: Red grape, lipids, lipoproteins, homocystein, athersclerosis.

\section{INTRODUCTION}

Consumption of grape flavonoids has been shown to confer antioxidant protection, inhibit platelet activity, reduce thrombus formation and lead to the concentration of inflammatory biomarkers [1, 2]. This effect may be considered to be beneficial for the prevention of cardiovascular disease [1]. In vitro studies showed that grape juice has significant antioxidant activity and can inhibit oxidation of low density lipoprotein (LDL) [1,2]. Also, red grape juice $(\mathrm{RGj})$ supplementation improves the lipoprotein profile by decreasing plasma concentrations of LDL and increasing those of high density lipoprotein (HDL) and greatly reducing the plasma concentrations of oxidized LDL [1]. Apolipoproteine B (apo B) is required for lipoprotein synthesis and secretion [3]. The catabolism of LDL that depends on the recognition of cellular receptors by apo B allows researchers to assess the risk of cardiovascular disease [4].

On the other hand, RGj consumption is associated with significantly lower cholesterol/HDL as atherogenic index, which is commonly used as the best lipid parameter for determining human heart disease risk [5]. Recently, metaanalysis of four large prospective studies has showed that for about $2 \%$ increase from an individual with normal high density lipoprotein-cholesterol (HDL-C) level, the incidence of coronary events decreases by $2 \%$ in men and $3 \%$ in

*Address correspondence to this author at the Department of Clinical Biochemistry and Nutrition, Faculty of Medicine, Urmia University of Medical Sciences, Urmia, Iran; Tel: +98-9141415879; Fax: +98-4412780801; E-mail: mhansari1@ hotmail.com women [6]. An increase in HDL-C is mainly due to an HDL3 subfraction increase. However, Rakic et al., reported that increases in apo AI and apo AII occur in HDL3 and HDL2 subfractions and that the changes in apo AI and apo AII were comparable and parallel to those of HDL-C [7].

The newest independent risk factor for cardiovascular disease is excess levels of homocysteine (Hcy) [8]. Hcy, a non-protein-forming sulfur amino acid, is an intermediate in methionine metabolism [9]. Although severe hyperhomocysteinemia is rare among the general population, mild hyperhomocysteinemia has been reported in $7 \%$ of the general population and up to $30 \%$ of patients with coronary and peripheral vascular disease [8]. Levels only $12 \%$ above the upper limit of normal plasma Hcy are associated with a 3 -fold increase in the risk of acute myocardial infarction [8, 10]. Studies reported that grape juices and grape skin extracts could prevent Hcy-induced endothelial dysfunction [11].

The aim of this study was to evaluate the effects of Iranian RGj consumption on HDL-C, apo $\mathrm{AI}$, apoB and Hcy levels in healthy human volunteers.

\section{MATERIALS AND METHODS}

Twenty six healthy and nonsmoking volunteers males aged 25-60 years, who were taking no medication, were asked to consume $150 \mathrm{ml}$ of pure RGj twice per day for one month. The RGj used in this project provided by UromNarin Co. (Urmia, Iran) and contained a total antioxidant capacity of $9.6 \mathrm{mmol} / \mathrm{kg}$ and $19.09 \mathrm{mg} / \mathrm{dl}$ of phenolic compounds (analyzed by Chemical Laboratories for Food Stuff-WaterEnvironmental Analysis, Germany). All volunteers 
continued their habitual diets during the study. Blood samples were drawn after fasting for 12 hours before study entry and after one month of RGj consumption. Blood was collected and allowed to clot for 15 minutes at room temperature and then centrifuged at $4000 \mathrm{rpm}$ in a bench centrifuge for 10 minutes. For Hcy assessment, blood was collected in tubes containing EDTA- $\mathrm{K}_{2}(1 \mathrm{mg} / \mathrm{ml})$ and then plasma samples were separated. Serum and plasma samples were immediately stored at $-80^{\circ} \mathrm{C}$ until analysis.

HDL-C was measured by direct enzymatic method using automated chemical analyzer BT-3000 (Biotecknica instruments, Italy). Serum apo AI and apo B concentrations were measured by nephelometric methods involving a reaction with specific antiserum to form an insoluble complex using mini nephelometric instruments (Binding Site Co., UK). Total plasma Hcy levels were measured by enzyme immunoassay (EIA) method using an EIA kit (AxisShield Diagnostic Co., UK).

Statistical analysis were performed by student's paired ttests and results are given as means \pm SD. Statistical significance was accepted as $\mathrm{p}<0.05$.

\section{RESULTS}

Figures show the serum HDL-C, apo AI, apo B and plasma Hcy levels before and after one month $\mathrm{RGj}$ consumption. HDL-C values were $41.44 \pm 4.50 \mathrm{mg} / \mathrm{dl}$ and $44.37 \pm 4.30 \mathrm{mg} / \mathrm{dl}$ before and after $\mathrm{RGj}$ consumption, respectively $(\mathrm{p}<0.0001$; Fig. 1). Serum apo B levels measured $149.0 \pm 22.35 \mathrm{mg} / \mathrm{dl}$ and $157.19 \pm 18.60 \mathrm{mg} / \mathrm{dl}$ before and after $R G j$ consumption, respectively $(\mathrm{p}<0.002$; Fig. 2). Serum apo AI measured $154.27 \pm 21.55 \mathrm{mg} / \mathrm{dl}$ and $155.35 \pm 21.07 \mathrm{mg} / \mathrm{d}$ l before and after RGj consumption, respectively, which show that it was not changed significantly ( $>00.05 ;$ Fig. 3). Plasma Hcy levels were $7.70 \pm$ $2.80 \mu \mathrm{mol} / \mathrm{l}$ and $6.20 \pm 2.30 \mu \mathrm{mol} / \mathrm{l}$ before and after $\mathrm{RGj}$ consumption, respectively, thus indicating a significantly decrease ( $<<0.001 ;$ Fig. 4).

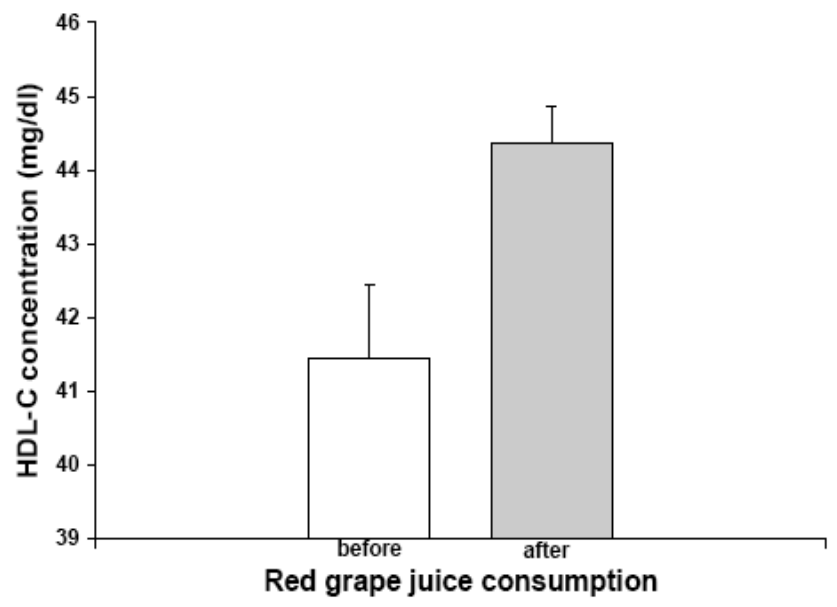

Fig. (1). HDL-C concentration before and after red grape juice consumption $(\mathrm{p}<0.0001)$.

\section{DISCUSSION}

The protective effects of fruits and vegetables against coronary heart disease (CHD) and myocardial infarction have been reported in numerous populations worldwide [12].

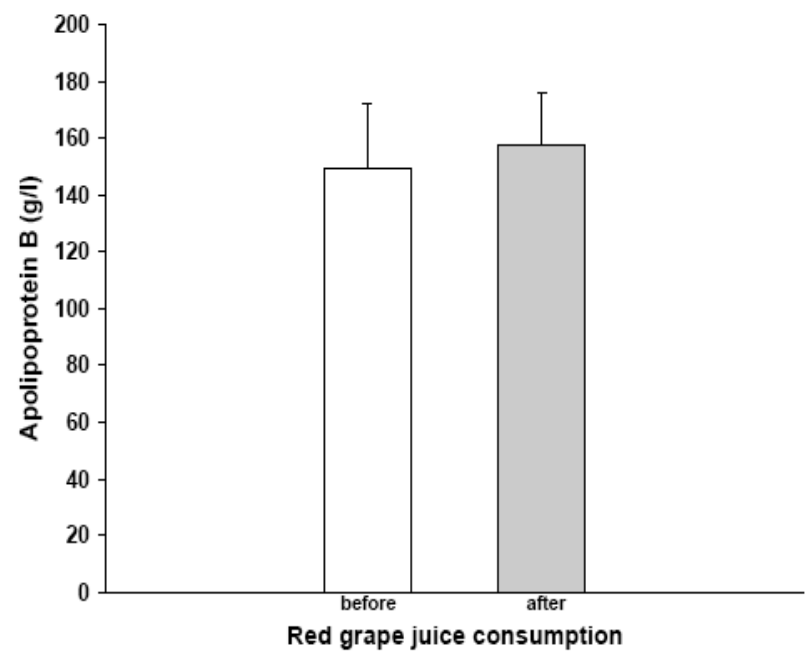

Fig. (2). Apolipoprotein B concentration before and after red grape juice consumption $(\mathrm{p}<0.002)$.

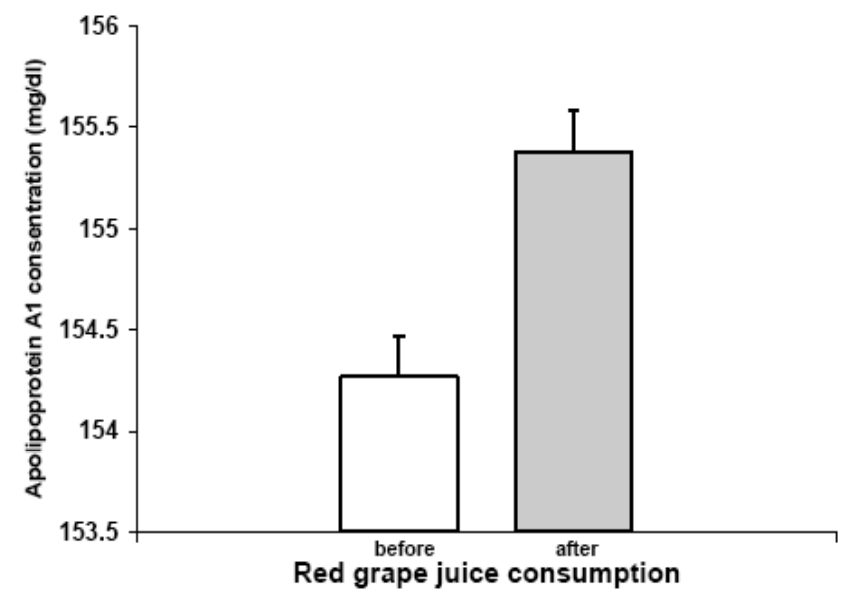

Fig. (3). Apolipoprotein AI concentration before and after red grape juice consumption ( $p>0.05$ ).

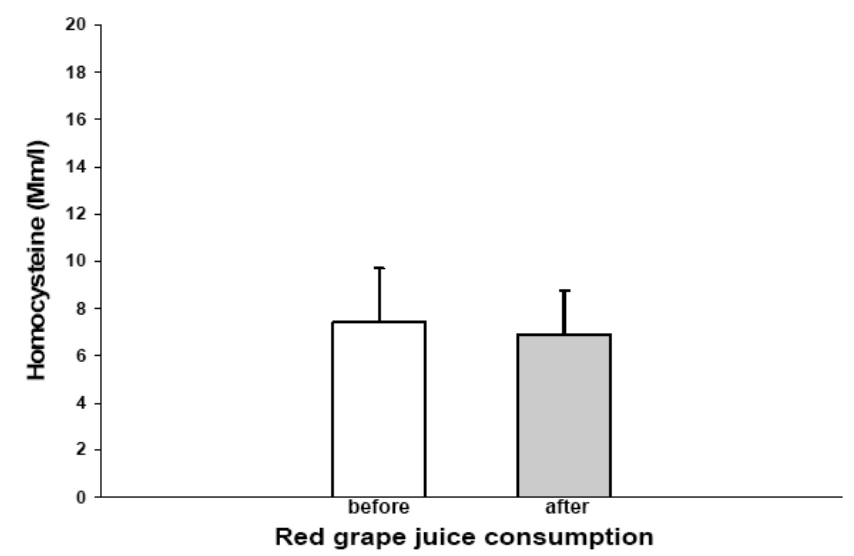

Fig. (4). Homocysteine concentration before and after red grape juice consumption $(\mathrm{p}<0.001)$.

Studies on the Mediterranean and French diets show that low rates of cardiovascular disease occur despite diets being rich in saturated fat and cholesterol [13]. The Mediterranean diet is rich in polyphenolics from vegetables and fruits, 
especially red grape, and these components are likely to confer some cardioprotective effects [13-15]. The Lyon heart study showed a $76 \%$ reduction in clinical events in subjects consuming a Mediterranean diet [13, 16]. For some individuals, dietary and lifestyle modification may be all that is needed to prevent CHD [17]. The bioavailability of dietary polyphenols, including those from grape derivatives, has been shown previously by many authors [18]. Ingestion of concentrated $\mathrm{RGj}$ as a polyphenol-rich dietary supplement exerts hypolipidemic, antioxidant and anti-inflammatory effects in healthy subjects [1]. Purple grape juice consumption improves endothelial function and reduces the susceptibility of LDL cholesterol to oxidation in patients with coronary artery disease [19].

Castilla et al., showed RGj supplementation is associated with parallel reductions in the concentrations of both LDL-C and apo B, and increases in HDL-C and apo AI [1]. However, Coimbra et al., reported no effect on apo B, apo AI and HDL-C levels after consumption of purple grape juice in healthy individuals for 14 days [20]. Auger et al., clearly demonstrated that consumption of polyphenol extract of $\mathrm{RGj}$ reduced plasma apo $\mathrm{B}$ concentration with no effect on apo AI [4]. Although, we show that RGj supplementation increase apo B and increase HDL-C levels but do not have a significant effect on apo AI concentration. This discrepancy may be related to differences in the composition and concentration of polyphenols in the $\mathrm{RGj}$ concentration in different studies. A polyphenol level of $19.09 \mathrm{mg} / \mathrm{dl}$ in the present work compares with that of $644 \mathrm{mg} / \mathrm{dl}$ in Castilla et al., study.

HDL-C is known to be a significant and independent predictor of CHD risk [6]. A significant increase found in plasma levels of HDL-C in subjects receiving $\mathrm{RGj}$ for 14 days [21, 22]. However, Aminian et al. showed that unripe grape juice consumption had no incremental effect on serum HDL-C levels in healthy individuals [17].

Hcy is an independent risk factor for vascular disease [9] and high circulating Hcy concentration is associated with an increased risk of occlusive vascular disease [8, 23]. Many studies indicate that Hcy-induced vascular injury involves oxidative stresses. Hcy is able to produce $\mathrm{O}_{2}{ }^{-}$and other free radicals by autoxidation in the presence of metals. Our study showed that RGj consumption lowers Hcy levels. Lower Hcy concentrations may introduce another possible mechanism for the vascular protective effects in populations that consume $\mathrm{RGj}$.

To our knowledge, no other study has investigated the direct effects of $\mathrm{RGj}$ consumption on serum Hcy levels, and in this sense, our work was the first study to show that $R G j$ consumption decreases Hcy levels and may be helpful to prevent CVD.

\section{ACKNOWLEDGEMENTS}

This research was supported by a grant from Sarouneh laboratory research units. We are grateful to Sarouneh stuffs in helping this research work.

\section{REFERENCES}

[1] Castilla, P.; Echarri, R.; Davalos, A.; Cerrato, F.; Ortega, H.; Teruel, J.L.; Lucas, M.F.; Gomez-Coronado, D.; Ortuno, J.; Lasuncion, M.A. Concentrated red grape juice exerts antioxidant, hypolipidemic, and antiinflammatory effects in both hemodialysis patients and healthy subjects. Am. J. Clin. Nutr., 2006, 84, 252-262. O'Byrne, D.J.; Devaraj, S.; Grundy, S.M.; Jialal, I. Comparison of the antioxidant effects of Concord grape juice flavonoids alphatocopherol on markers of oxidative stress in healthy adults. Am. $J$. Clin. Nutr., 2002, 76, 1367-1374.

[3] Davalos, A.; Fernandez-Hernando, C.; Cerrato, F.; Martinez-Botas, J.; Gomez-Coronado, D.; Gomez-Cordoves, C.; Lasuncion, M.A. Red grape juice polyphenols alter cholesterol homeostasis and increase LDL-receptor activity in human cells in vitro. J. Nutr., 2006, 136, 1766-1773.

[4] Auger, C.; Caporiccio, B.; Landrault, N.; Teissedre, P.L.; Laurent, C.; Cros, G.; Besancon, P.; Rouanet, J.M. Red wine phenolic compounds reduce plasma lipids and apolipoprotein B and prevent early aortic atherosclerosis in hypercholesterolemic golden Syrian hamsters (Mesocricetus auratus). J. Nutr., 2002, 132, 1207-1213.

[5] Vinson, J.A.; Teufel, K.; Wu, N. Red wine, dealcoholized red wine, and especially grape juice, inhibit atherosclerosis in a hamster model. Atherosclerosis, 2001, 156, 67-72.

[6] Eccleston, C.; Baoru, Y.; Tahvonen, R.; Kallio, H.; Rimbach, G.H.; Minihane, A.M. Effects of an antioxidant-rich juice (sea buckthorn) on risk factors for coronary heart disease in humans. J. Nutr. Biochem., 2002, 13, 346-354.

[7] Belleville, J. The French paradox: possible involvement of ethanol in the protective effect against cardiovascular diseases. Nutrition, 2002, 18, 173-177

[8] Fu, W.; Conklin, B.S.; Lin, P.H.; Lumsden, A.B.; Yao, Q.; Chen, C. Red wine prevents homocysteine-induced endothelial dysfunction in porcine coronary arteries. J. Surg. Res., 2003, 115, 82-91.

[9] Jacques, P.F.; Bostom, A.G.; Wilson, P.W.; Rich, S.; Rosenberg, I.H.; Selhub, J. Determinants of plasma total homocysteine concentration in the Framingham Offspring cohort. Am. J. Clin. Nutr., 2001, 73, 613-621.

[10] Chen, C.; Conklin, B.S.; Ren, Z.; Zhong, D.S. Homocysteine decreases endothelium-dependent vasorelaxation in porcine arteries. J. Surg. Res., 2002, 102, 22-30.

[11] Flesch, M.; Schwarz, A.; Bohm, M. Effects of red and white wine on endothelium-dependent vasorelaxation of rat aorta and human coronary arteries. Am. J. Physiol., 1998, 275, H1183-1190.

[12] Houston, M.C.; Cooil, B.; Olafsson, B.J.; Raggi, P. Juice Powder Concentrate and Systemic Blood Pressure, Progression of Coronary Artery Calcium and Antioxidant Status in Hypertensive Subjects: A Pilot Study. Evid Based Complement Alternat. Med., 2007, 4, 455462.

[13] Pal, S.; Ho, N.; Santos, C.; Dubois, P.; Mamo, J.; Croft, K.; Allister, E. Red wine polyphenolics increase LDL receptor expression and activity and suppress the secretion of ApoB100 from human HepG2 cells. J. Nutr., 2003, 133, 700-706.

[14] Hertog, M.G.; Kromhout, D.; Aravanis, C.; Blackburn, H.; Buzina, R.; Fidanza, F.; Giampaoli, S.; Jansen, A.; Menotti, A.; Nedeljkovic, S.; et al. Flavonoid intake and long-term risk of coronary heart disease and cancer in the seven countries study. Arch. Intern. Med., 1995, 155, 381-386.

[15] Zern, T.L., Fernandez, M.L. Cardioprotective effects of dietary polyphenols. J. Nutr., 2005, 135, 2291-2294.

[16] De Lorgeril, M.; Salen, P.; Martin, J.L.; Mamelle, N.; Monjaud, I.; Touboul, P.; Delaye, J. Effect of a mediterranean type of diet on the rate of cardiovascular complications in patients with coronary artery disease. Insights into the cardioprotective effect of certain nutriments. J. Am. Coll. Cardiol., 1996, 28, 1103-1108.

[17] Aminian, B.; Massoompour, S.M.; Sadeghalvaad, A.; Omrani, G.H. Unripe grape juice (verjuice) as a lipid-lowering agent: fact or fiction. Arch. Iranian Med., 2003, 6(1), 32-34.

[18] Nigdikar, S.V.; Williams, N.R.; Griffin, B.A.; Howard, A.N. Consumption of red wine polyphenols reduces the susceptibility of low-density lipoproteins to oxidation in vivo. Am. J. Clin. Nutr. 1998, 68, 258-265.

[19] Stein, J.H.; Keevil, J.G.; Wiebe, D.A.; Aeschlimann, S.; Folts, J.D. Purple grape juice improves endothelial function and reduces the susceptibility of LDL cholesterol to oxidation in patients with coronary artery disease. Circulation, 1999, 100, 1050-1055.

[20] Coimbra, S.R.; Lage, S.H.; Brandizzi, L.; Yoshida, V.; da Luz, P.L. The action of red wine and purple grape juice on vascular reactivity is independent of plasma lipids in hypercholesterolemic patients. Braz. J. Med. Biol. Res., 2005, 38, 1339-1347. 
[21] Albers, A.R.; Varghese, S.; Vitseva, O.; Vita, J.A.; Freedman, J.E. The antiinflammatory effects of purple grape juice consumption in subjects with stable coronary artery disease. Arterioscler. Thromb. Vasc. Biol., 2004, 24, e179-180.

[22] Castilla, P.; Davalos, A.; Teruel, J.L.; Cerrato, F.; FernandezLucas, M.; Merino, J.L.; Sanchez-Martin, C.C.; Ortuno, J.; Lasuncion, M.A. Comparative effects of dietary supplementation with red grape juice and vitamin $\mathrm{E}$ on production of superoxide by circulating neutrophil NADPH oxidase in hemodialysis patients. Am. J. Clin. Nutr., 2008, 87, 1053-1061.

[23] Dixon, J.B.; Dixon, M.E.; O'Brien, P.E. Reduced plasma homocysteine in obese red wine consumers: a potential contributor to reduced cardiovascular risk status. Eur J. Clin. Nutr., 2002, 56, 608-614.

Received: March 20, 2010

Revised: June 28, 2010

Accepted: July 10, 2010

(c) Khadem-Ansari et al.; Licensee Bentham Open.

This is an open access article licensed under the terms of the Creative Commons Attribution Non-Commercial License (http://creativecommons.org/licenses/by-nc/3.0/) which permits unrestricted, non-commercial use, distribution and reproduction in any medium, provided the work is properly cited. 